Journal of Computer Science 6 (3): 250-252, 2010

ISSN 1549-3636

(C) 2010 Science Publications

\title{
Development of a "Programmable Logic Controller Circuitry" For Optimal Power Distribution in a Manufacturing Industries
}

\author{
K. Selvaraj \\ Department of Electrical and Electronics Engineering, \\ Sri Sapthagiri Institute of Technology, Ocheri Vellore Dist, Tamilnadu, South India
}

\begin{abstract}
Problem statement: In industries the powder demand/consumption is fixed by the power supply agency. However, at times, the demand/Consumption may exceed the fixed level and will result in, the industry paying heavy penalties to the supply agency. In most of the large and medium scale industries, controlling of power consumption is done by monitoring the powder demand indicator unit activated by a microprocessor $(\mu p)$ circuitry. The major drawback of controlling the consumption using $\mu p$ circuitry is that the peak value is fixed and cannot be changed in proportion to the fluctuation in load. To address above issue an attempt is made in the present work, to replace the $\mu$ p circuitry with a PLC circuitry which provides for flexibility, i.e., to change maximum demand level based on the fluctuations in the load through suitable programming in the PLC circuitry. Approach: A detailed literature review was made on the application and functioning of supply controls using $\mu$ p circuitry along with the associated limitations. The application of PLC circuitry in the place of $\mu$ p circuitry is reviewed. The methodology of the present research was presented. The experimental work was briefed. Results: The various results were listed and discussed. Major conclusions drawn, based on the present research was shown. Conclusion: The major contribution of the present study lies, in zeroing the frequency of the power consumption, crossing the fixed level, through the design and development of an optimal PLC circuitry resulting in a significant savings in the power consumption.
\end{abstract}

Key words: Microprocessor, programmable logic controller, demand level, flexibility, automation

\section{INTRODUCTION}

In large and medium scale industries, the physically implemented power demand indicator is programmed using microprocessors. The major setback in the functioning of the demand indicator using microprocessor circuitry is that it runs on an unchangeable programme, thereby, the once fixed demand level cannot be altered suiting the requirements of the fluctuations in loads. To address above issues a program PLC is proposed in the place of a microprocessor circuitry. This has provided for flexibility in changing the maximum demand level suiting to the fluctuations in the load. PLC is a controlling device consisting of a programmable processing unit using specialized computer languages. Typically the programme is written in a development environment on a personal computer and is downloaded on to a PLC directly through a cable. The programme is stored in a non-volatile memory of the PLC. The PLC circuitry monitors the status of multiple sensor inputs, which control the output actuators like motor starters solenoids. A single PLC circuitry controls all the above individual output actuators. PLC circuitry offers a wide range of functions including relay controls, motor controls, process controls, complex networks as well as distributed control systems. In the present work a PLC circuitry is developed relating to power distribution. The developed PLC identifies total power from the supply unit and controls the distribution of the same to the manufacturing and non-manufacturing areas of the industry during the periods of demand exceeding a certain level. The PLC partly shut down the supply in the non-manufacturing area.

Literature review: In early 20th century, microprocessors are used for controlling power distribution among various units of an industry (Hughes, 2000). The applications of microprocessor suffer from a basic limitation that it fails to alter the maximum demand level during load fluctuations (Rosin, 2000; Berger, 2003). This has resulted in development of PLC circuitry to be incorporated in the place of microprocessor, in order that the demand level can be altered to suit the load fluctuations resulting in a smooth uninterrupted and continuous power distribution (Jack, 2005). PLCS are miniature individual computers (Siemens AG, 2003a), using hardware and software to perform the control functions. 
Table 1: PLCS in comparison with microprocessor controls

\begin{tabular}{lll}
\hline PARTS & $\mu \mathrm{C}$ & PLC \\
\hline Inputs & $3-5 \mathrm{~V}$ & Higher rating \\
Input condition & $\begin{array}{l}\text { Non-isolated I/O } \\
\text { Programming }\end{array}$ & $\begin{array}{l}\text { Programming of } \mu \mathrm{C} \\
\text { Isolated I/O }\end{array}$ \\
$\begin{array}{ll}\text { Programming of } \\
\text { Environment }\end{array}$ & $\begin{array}{l}\mu \mathrm{C} \text { is affected by noise } \\
\text { Outputs }\end{array}$ & $\begin{array}{l}\text { Fast is easy } \\
\text { Noise can't affected the PLC } \\
\text { because of disturbance }\end{array}$ \\
\end{tabular}

The PLCS consist of two basic sections namely CPU and the Input/Output interface systems (Siemens AG, 2003a). Various controllers that are normally used are microprocessors, proportional interface derivative controls (Siemens AG, 2003b). The major disadvantages of the controls are, the problem of fixed imaginary values used for comparing and controlling. PLCS are preferred more for their merit of providing flexible and absolute real values. The first PLC was traced back to (Hughes, 2000) when Bed Ford Associate developed a device called modular digital control for general motors. This was used by General Motors to replace the traditional relay based machine control system. Today's PLCS are designed not only to communicate with control systems, but also to perform reporting functions and diagnose the failures (Siemens AG, 2003c). Table 1 brings out a comparison between the applications of microprocessors and PLCS.

\section{MATERIALS AND METHODS}

In operation of CPU part the PLC circuitry reads data from connected field devices through the use of its input interfaces and then executes control programme that has been stored in its memory system. The programmes are typically executed in ladder logic language that closely resembles a relay-based wiring schematic and is executed in the CPU's memory, prior to operation. Finally, based on the programme the PLC controls or updates output devices through the output interfaces. This process, also known as scanning, continues in the same sequence without interruption and changes only, when a change is made in the PLC circuitry controller (Rosin, 2000; Berger, 2003). This successfully controls the maximum power consumed during a given period of time. The developed PLC monitors the power used and turns off non essential loads during the period of higher power use. The methodology consist of using a PLC controller device that controls the supply when demand approaches a preset value by switching off all nonessential loads (OPC Foundation, 2003).

\section{Programming languages used in PLC circuitry:}

- Ladder diagram language

- Boolean language
- Functional chart system

- Instruction list

- $\quad$ Structural list

Of the 5 languages listed above, ladder diagram language is used for its simplicity and ease of execution (Siemens AG, 2004).

Principle of working of the maximum demand indicator: The PLC module used in the input/output system connects field input devices of the ON/OFF nature of the selector switches. It senses the signal received from the sensors and converts the input signals to the correct voltage level for the PLC circuitry and finally controls the maximum demand indicator for optimal distribution of power.

Experimental work: A medium scale processing industry is considered for the present investigation. The maximum power demand as fixed by supply agency is $75 \mathrm{~kW}$. The industry consists of the following power consuming areas:

- $\mathrm{O}_{1}$-Manufacturing Area 1(MA1) rated $40 \mathrm{kw}$

- $\mathrm{O}_{2}$-Manufacturing Area 2 (MA2) rated $40 \mathrm{kw}$

- $\mathrm{O}_{3}$-General area $1(\mathrm{NMA} 1)$ rated $2.5 \mathrm{kw}$

- $\mathrm{O}_{4}$-General area 2(NMA2) rated $2.5 \mathrm{kw}$

- $\mathrm{O}_{5}$-Tripped off lights (negligible rating)

- $\mathrm{O}_{6-}$ Controller running (negligible rating)

- $\mathrm{O}_{7}$-Wooter (negligible rating)

The maximum demand indicator and controller is supplied with $24 \mathrm{~V}$ input. The PLC used is the CB2o PLC of $124-6 \times 100 \mathrm{~mm}$ dimensions. The PLC includes 12 inputs in which 6 analogue and 8 relay type outputs.

To indicate the running condition of the PLC the output 06 always glows. The manufacturing areas 1 and 2 include core area of industry which requires uninterrupted power supply.

A Wattmeter is connected to indicate the increase in power demand level namely $75 \mathrm{~kW}$. After exceeding $75 \mathrm{~kW}$ the PLC receives signals from the rheostat to trip off the general area. The excession in the power does not last more than fraction of a millisecond. Hence a short time trip off can bring about a very effective power control. Figure 1 shows the variation in power demand into the various machines switched on in the manufacturing and non manufacturing areas for a period of $1 \mathrm{~h}$ on a particular day of investigation.

When some of the sensing units like Wattmeter fail, the PLC will detect the Wattmeter failure and give the alarm. 


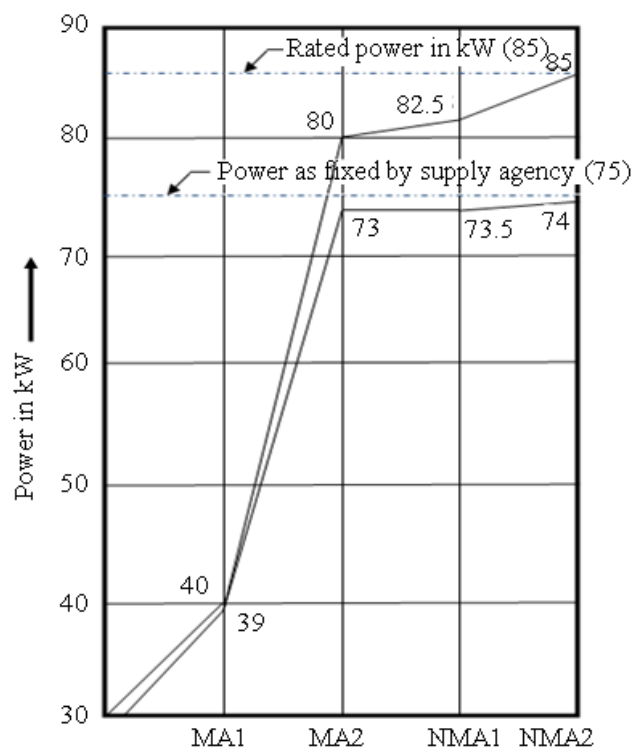

Fig. 1: Power graph analysis with PLC

Table 2: Variations in rated and actual consumed power

\begin{tabular}{lrrrr}
\hline Area & 1st $\mathrm{h}$ & 2nd $\mathrm{h}$ & $3 \mathrm{rd} \mathrm{h}$ & 4 th $\mathrm{h}$ \\
\hline Manufacturing area 1/Rated power $(\mathrm{kW})$ & 40.0 & 40.0 & 40.0 & 40.0 \\
Manufacturing area 1/actual & 39.0 & 38.0 & 35.0 & 36.0 \\
power utilized $(\mathrm{kW})$ & & & & \\
Manufacturing area 2/rated power $(\mathrm{kW})$ & 40.0 & 40.0 & 40.0 & 40.0 \\
Manufacturing area 2/actual power $(\mathrm{kW})$ & 34.0 & 34.0 & 37.0 & 37.0 \\
Non manufacturing area 1/rated power $(\mathrm{kW})$ & 2.5 & 2.5 & 2.5 & 2.5 \\
Non manufacturing area 1/actual power $(\mathrm{kW})$ & 0.5 & 1.0 & 1.0 & 0.5 \\
Non manufacturing area 2/rated power $(\mathrm{kW})$ & 2.5 & 2.5 & 2.5 & 2.5 \\
Non manufacturing area 2/actual power $(\mathrm{kW})$ & 0.5 & 1.0 & 1.0 & 0.5 \\
Maximum value of controlled power $(\mathrm{kW})$ & 74.0 & 74.0 & 74.0 & 74.0 \\
\hline
\end{tabular}

Table 2 gives the fluctuation of power demand and the function of the PLC to control the load in different units resulting in zeroing the frequency of the maximum power consumed exceeding the preset value of $75 \mathrm{~kW}$.

\section{RESULTS AND DISCUSSION}

Table 2 gives the details pertaining to the rated power and the demand power as fixed by the supplier with respect to time on a particular day of investigation using PLC circuitry. It is seen that the corrective action is automatic and instantaneous and demand exceeding the preset value is zeroed down:

- If the PLC circuitry is replaced by the $\mu$ p circuitry an increase in demand over and above the preset value would have occurred for a considerable period of time

- Since an inbuilt automatic rectification is provided with PLC circuitry, it is observed that the life of the machines in the manufacturing areas 1 and 2 is considerably increased in a given 1 year investigation period

- It is also noticed from Table 2 that the overall saving in power (as a result of shedding down non manufacturing areas) is about $6 \%$ in a given period of time

\section{CONCLUSION}

Based on above results the following major conclusions are drawn:

- The overall tariff rate for the industry under investigation, even when the demand exceeds the fixed preset value, is considerably low as a result of power shedding in non manufacturing areas.

- The life of the machine in the manufacturing area is considerably increased.

- A large scale power saving, over a given period of time is seen.

- A larger flexibility in programming using PLC circuitry is noticed.

\section{REFERENCES}

Berger, H., 2003. Automating with SIMATIC: Integrated Automation with SIMATIC S7-300/400: Controllers, Software, Programming, Data Communication, Operator Control and Process Monitoring. 2nd Edn., Wiley-VCH, ISBN: 3895782238, pp: 221.

Hughes, T.A., 2000. Programmable Controllers. 3rd Edn., The Instrumentation, Systems, and Automation Society, pp: 334.

Jack, H., 2005. Automating manufacturing systems with PLCs. http://webbooks.net/freestuff/plc.pdf

OPC Foundation, 2003. OPC Foundation homepage. http://www.opcfoundation.org

Rosin, A., 2000. Programmable controllers Simatic S7. Master Thesis, Tallinn, TTU, Estonian, pp: 120.

Siemens AG, 2003a. Siemens LOGO Manual. http://www.control.com/thread/1254143982

Siemens AG, 2003b. SIMATIC S7-200. Programmable Controller System http://cmsapps.sea.siemens.com/controls/icc/06Ind Control_\%20pdfs/06IC_15/15_03-04.pdf

Siemens AG, 2003c. SIMATIC HMI WinCC. Configuration Manual. http://www.pacontrol.com/siemensmanuals/Simatic-HMI-WinCC_Basics.pdf

Siemens AG, 2004. SIMATIC Programming with $\begin{array}{llll}\text { STEP } & 7 \text { Lite } 3.0 . & \text { Manual. }\end{array}$ http://legacy.solar.dk/upload/downloads/industri/S 7LiteV30_e.pdf 